\title{
Macroscopic Pseudoexfoliation Material within the Iridocorneal Angle
}

Berry EC ${ }^{1 *}$, Mullany $\mathrm{S}^{1}$, Siggs $\mathrm{OM}^{1,2}$ and Craig J E ${ }^{1}$ ${ }^{1}$ Department of Ophthalmology, Flinders University, Australia

${ }^{2}$ Garvan Institute of Medical Research, Australia

*Corresponding author: Berry EC, Department of Ophthalmology, Flinders University, 1Flinders Drive, Bedford Park SA 5042, Australia

Received: August 27, 2021; Accepted: September 11, 2021; Published: September 18, 2021

\section{Clinical Image}

During routine follow-up of an 85-year-old man with bilateral pseudoexfoliative glaucoma, unusually large newly deposited macroscopic sheets of pseudoexfoliation material were observed in the right inferior iridocorneal angle (Panel A). These sheetlike accumulations were further characterized as hyper-reflective filamentous structures on anterior segment optical coherence tomography (Panel B). Pseudoexfoliative glaucoma had been diagnosed four years prior with intraocular pressures of $34 \mathrm{mmHg}$ $\mathrm{OD}$ and $28 \mathrm{mmHg} \mathrm{OS}$, and vertical cup-to-disc ratios of $0.9 \mathrm{OD}$ and 0.85 OS. Intraocular pressure (IOP) was initially managed with topical latanoprost and timolol OU, followed by trabeculectomy OD. With

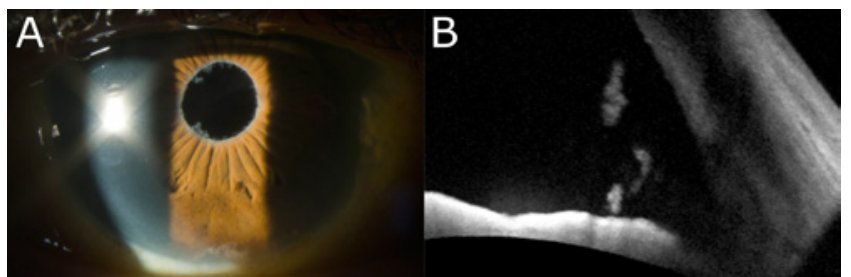

Figure 1:

continued topical therapy, his IOP remained well controlled for three years, after which selective laser trabeculoplasty OD was performed due to increasing IOP and severe visual field loss. Pseudoexfoliation syndrome is a systemic disease often associated with glaucoma due to accumulation of pseudoexfoliation material within the trabecular meshwork, and consequent impairment of aqueous outflow (Figure 1).

\section{Acknowledgements}

We thank Angela Chappell and Carly Emerson (Flinders Medical Centre) for ophthalmic imaging. 